\title{
Degradación de la Clorofila Presente en (Spirogyra spp.) como Indicator Socio Ambiental de la Presencia de Plaguicidas organofosforados
}

\author{
González García J. C.,
}

Magister en Protección Ambiental, Profesor a Tiempo Completo de la

Escuela Superior Politécnica de Chimborazo, Ecuador

Silva Quiguiri N. A.,

Ingeniera en Biotecnología Ambiental, Investigadora Colaboradora de la

Escuela Superior Politécnica de Chimborazo, Ecuador

Lindao Córdova V. A.,

Doctor en Ciencias Ambientales de la Universidad Nacional San Marcos, Lima Perú, Profesor Tiempo Completo de la Escuela Superior Politécnica de Chimborazo, Ecuador

Godoy Ponce S. C.,

Magister en Gestión del Desarrollo Local Comunitario, Profesor a Tiempo

Completo de la Escuela Superior Politécnica de Chimborazo, Ecuador

Medina Serrano C. A.,

Master Universitario en Química Aplicada a la Investigación y a la Industria,

Profesor a Tiempo Completo de la Escuela Superior Politécnica de

Chimborazo, Ecuador

Doi:10.19044/esj.2019.v15n21p395 URL:http://dx.doi.org/10.19044/esj.2019.v15n21p395

\section{Resumen}

En la presente investigación se estudió la alteración de la clorofila presente en Spirogyra spp. como indicador de la presencia de tres plaguicidas organofosforados: Curacrón, Malatión y Acefato, a través de ensayos realizados a diferentes concentraciones de los plaguicidas durante un lapso de 72 horas de exposición. Transcurrida la etapa experimental se pudo observar la pérdida de la coloración verde de las algas como resultado de la alteración de su pigmentación natural. El análisis de varianza realizado a los resultados de la medición de la absorbancia de las muestras demuestra una disminución de la concentración de clorofila, en comparación con las muestras testigo de los ensayos. La sensibilidad de las algas al ser expuestas a los plaguicidas organofosforados es una manifestación del alto potencial bioindicador de Spirogyra spp. Este estudio brinda a los agricultores de la comunidad de San Antonio la posibilidad de detectar de manera temprana la presencia de 
plaguicidas en sus fuentes de agua y abre las puertas a la investigación para posteriores aplicaciones.

Palabras clave: Spirogyra spp., Clorofila, Plaguicidas organofosforados, Malatión, Curacrón, Acefato

\title{
Degradation of Chlorophyll Present (Spirogyra spp.) as an Environmental Partner Indicator of the Presence of Organophosphorus Pesticides
}

\author{
González García J. C., \\ Magister en Protección Ambiental, Profesor a Tiempo Completo de la \\ Escuela Superior Politécnica de Chimborazo, Ecuador
}

Silva Quiguiri N. A.,

Ingeniera en Biotecnología Ambiental, Investigadora Colaboradora de la

Escuela Superior Politécnica de Chimborazo, Ecuador

Lindao Córdova V. A.,

Doctor en Ciencias Ambientales de la Universidad Nacional San Marcos, Lima Perú, Profesor Tiempo Completo de la Escuela Superior Politécnica de

Chimborazo, Ecuador

Godoy Ponce S. C.,

Magister en Gestión del Desarrollo Local Comunitario, Profesor a Tiempo

Completo de la Escuela Superior Politécnica de Chimborazo, Ecuador

\section{Medina Serrano C. A.,}

Master Universitario en Química Aplicada a la Investigación y a la Industria,

Profesor a Tiempo Completo de la Escuela Superior Politécnica de

Chimborazo, Ecuador

\begin{abstract}
The alteration of the chlorophyll present in Spirogyra spp. was studied as an indicator of the presence of three organophosphorus pesticides, Curacron, Malathion and Acephate, with tests carried out at different concentrations of the pesticides during a period of 72 hours of exposure. After the experimental stage, it was possible to observe the loss of the green coloration of the algae as a result of the alteration of their natural pigmentation. The analysis of variance based on the results of the measurement of the absorbance of the samples demonstrated a decrease in the concentration of
\end{abstract}


chlorophyll compared to the control samples from the trials. The sensitivity of algae to being exposed to organophosphorus pesticides is a manifestation of the high bioindicator potential of Spirogyra spp., which provides farmers in the community of San Antonio the possibility of early detection of the presence of pesticides in their sources of water, and it also opens the doors to research for subsequent applications.

Keywords: Spirogyra spp., Chlorophyll, Organophosphorus pesticides, Malathion, Curacrón, Acephate

\section{Introducción}

En el Ecuador una de las actividades antropogénicas más contaminadoras es la agricultura, pues infinidad de productos agroquímicos son incorporados al suelo para controlar plagas y darle mayor fertilidad. Los plaguicidas, en su gran mayoría sintetizados por el hombre, se caracterizan por su alta toxicidad y por su baja degradabilidad, causando la pérdida de la biodiversidad de las zonas donde se realiza esa actividad (Orta, 2002).

Los plaguicidas, por su estabilidad estructural, tienden a biomagnificarse a través de las cadenas tróficas, bioacumulándose así en los organismos. Procesos naturales como: viento, precipitaciones, escorrentía superficial y filtración hacia los mantos freáticos pueden acarrear plaguicidas residuales, creando riesgos para el hombre y los sistemas ambientales (Orta, 2002).

Los plaguicidas organofosforados son ésteres orgánicos derivados del ácido fosfórico que se han convertido en los de mayor demanda por parte de los agricultores por su eficacia y bajo costo (Mariño, 2005).

Los pobladores de la comunidad de San Antonio del cantón Riobamba, Ecuador, desde hace más de diez años, cultivan tomate intensivamente bajo condiciones de invernadero, y para el control de plagas han aplicado plaguicidas de manera exagerada, siendo los más utilizados los organofosforados: Curacrón, Acefato y Malatión, los cuales serían la causa de serios problemas de contaminación del ambiente (Lindao, 2017).

Actualmente, para el diagnóstico y el control de la contaminación se utiliza el análisis químico, técnicas sofisticadas y equipos costosos. En el país, los análisis de plaguicidas residuales se realizan en laboratorios privados y de universidades, institutos de investigación y organismos gubernamentales competentes (Mariño, 2005).

Sin embargo, estas técnicas requieren de procesos largos y costosos, y en algunos casos pre-tratamientos, haciéndose necesario probar nuevos métodos para la detección temprana de contaminantes presentes en agua. En este contexto los bioindicadores son una alternativa eficaz $y$ 
comparativamente económica para detectar contaminantes para su posterior control.

Diversos organismos, entre ellos las algas, por su fisiología y morfología, han sido utilizados tradicionalmente como bioindicadores de contaminación, ya que manifiestan cambios físicos y químicos al ser expuestos a uno o varios contaminantes específicos. Las algas alertan a través de sus cambios sobre la presencia de contaminantes, para posteriormente tratarlos y evitar mayores problemas ambientales en el futuro.

Spirogyra spp., es un alga verde filamentosa, común en estanques y arroyos. Sus células son cilíndricas, más largas que gruesas, y unidas extremo con extremo en filamentos uniseriados, no ramificados, de longitud indefinida. Cada célula del alga tiene una gran vacuola central y uno a dos cloroplastos espirales y acintados con varios pirenoides (Cronquist, 1984).

Experimentos con Spirogyra spp. expuesta a diversas dosis de determinados plaguicidas de naturaleza organofosforada han dado como resultado una evidente alteración de su morfología, color y fisiología.

La pérdida de color por la degradación de la clorofila presente en las algas es una manifestación del contacto con plaguicidas, efecto que puede traducirse como un indicador de contaminación de agua, hábitat de las algas.

Conocer la sensibilidad de ciertos organismos al ser expuestos a sustancias tóxicas abre una nueva posibilidad en el campo de la prevención y posterior control de la contaminación, utilizando bioindicadores que siempre resultarán más económicos y prácticos.

\section{Materiales y métodos}

\section{Metodología}

\subsection{Metodología- fase de campo}

La toma de muestras de algas se realizó en la comunidad de San Antonio de la parroquia San Luis, cantón Riobamba, provincia de Chimborazo, Ecuador, sector agrícola por excelencia. Aquí se evidenció crecimiento de algas dentro de acequias y canales alimentados por descargas de agua provenientes del riego de cultivos aledaños, razón por la cual se decidió tomar muestras de biomasa algal de este lugar.

Se aplicó un muestreo simple utilizando la técnica de recolección y conservación de algas de agua dulce descrita por el Herbario de la Universidad de Panamá (Vergara, C. s.f.).

Para la identificación morfológica de Spirogyra spp. se aplicó la guía de campo para la identificación de algas, agricultura y agroalimentación de Canadá, propuesta por Nancy Sederiak y Mai-Linh Huynh, realizando una observación directa usando microscopio con un aumento de 40x y cámara digital incorporada para la captura de imágenes (Sederiak \& Huynh, 2006). 
Los plaguicidas utilizados en la experimentación fueron aquellos del grupo químico de los organofosforados, comercialmente conocidos como: Curacrón (O-(4-bromo-2-chlorophenyl)-O-ethyl S-propyl phosphorothioate), Acefato (O, S-Dimethyl acetylphosphoramidothioate) y Malatión (O, ODimethyl phosphorodithoate de diethyl mercaptosuccinate), que son los plaguicidas más utilizados según las encuestas aplicadas a los agricultores del sector (Lindao, 2017).

\subsection{Metodología-fase de laboratorio}

Una vez tomadas las muestras de algas se procedió a su adaptación y reproducción directa en 30 unidades experimentales en el laboratorio, donde se consideraron condiciones ambientales similares a las de la zona de proveniencia de las algas tales como: presencia de luz natural, temperatura aproximada de $17{ }^{\circ} \mathrm{C}$ y $\mathrm{pH}$ alrededor de 8 . Esta fase duró aproximadamente 21 días.

Las unidades experimentales consistieron en recipientes de plástico transparente de forma rectangular, cada uno con $10 \mathrm{~L}$ de agua, en donde se añadió 6,3 g de biomasa del alga, y donde posteriormente se analizó el efecto de los plaguicidas en la pigmentación de las algas.

Para satisfacer los requerimientos de macronutrientes de los cultivos se incorporó: nitrógeno, fósforo y potasio en una relación de $10 \mathrm{~g}$ de fertilizante soluble por cada $10 \mathrm{~L}$ de agua.

Una vez transcurrido el tiempo de adaptación y crecimiento de la biomasa en las unidades experimentales, se realizaron los ensayos para verificar el comportamiento de las algas al ser expuestas a tres plaguicidas a diferentes concentraciones. Se hicieron observaciones antes de añadir las sustancias, así como también 24, 48 y 72 horas después de la aplicación. La aplicación de los plaguicidas se realizó en tres dosis: la recomendada por el distribuidor (etiqueta), una dosis menor y otra mayor a la recomendada por la casa formuladora como se expresa en la Tabla 1.

Tabla 1. Tratamientos en estudio

\begin{tabular}{|ccccc|}
\hline Plaguicida & \multicolumn{3}{c}{ Ensayos } & Concentración \\
\hline \multirow{3}{*}{ Acefato } & A1a & A1b & A1c & 0,5 \\
& A2a & A2b & A2c & 1,0 \\
& A3a & A3b & A3c & 1,5 \\
\hline \multirow{3}{*}{ Malatión } & B1a & B1b & B1c & 1,5 \\
& B2a & B2b & B2c & 2,5 \\
& B3a & B3b & B3c & 3,5 \\
\hline \multirow{3}{*}{ Curacrón } & C1a & C1b & C1c & 0,29 \\
& C2a & C2b & C2c & 0,58 \\
& C3a & C3b & C3c & 0,87 \\
\hline
\end{tabular}


También se utilizaron 3 muestras testigo o blancos en el experimento (uno por cada plaguicida).

Durante la experimentación en laboratorio se controló el $\mathrm{pH}$, temperatura, conductividad y sólidos totales, se observaron los cambios de coloración en la biomasa de Spirogyra spp., y estos fueron verificados a través de microscopía óptica de 40x durante la duración del ensayo.

Finalmente se midió la absorbancia de la clorofila presente en las muestras al culminar la experimentación con la ayuda de un espectrofotómetro UV-visible en un rango de longitud de onda $(\lambda)$ entre 450 y $700 \mathrm{~nm}$ propios para determinación de clorofila.

\subsection{Análisis estadístico}

Se utilizó un diseño completamente al azar (DCA) con 3 plaguicidas, 3 dosis más el testigo y 3 repeticiones. Se determinó el coeficiente de variación y la prueba de Tukey al 5\% usando el programa INFOSTAT versión 2017 (Di Rienzo, 2017).

\section{Resultados}

Las condiciones ambientales que se controlaron mostraron poca variabilidad durante las 72 horas de experimentación, manifestándose incrementos de temperatura después del tercer día en especial en las unidades con el plaguicida Curacrón. El pH se mantuvo básico durante todos los ensayos con un valor alrededor de 8. A lo largo del experimento se observó un incremento en la conductividad eléctrica y sólidos totales por la adición tanto de nutrientes $(\mathrm{N}, \mathrm{Na}, \mathrm{K})$ como de los plaguicidas.

\subsection{Resultados Microscopía}

Los cambios físicos en las algas se determinaron de manera directa, y adicionalmente se realizaron observaciones al microscopio. Las tablas 2, 3, 4 y 5 muestran el registro fotográfico de los cambios de coloración manifestados por Spirogyra spp. tras la adición de los plaguicidas, en contraste con el testigo el cual mantuvo su pigmentación inicial. 
Tabla 2. Registro de fotografías de Spirogyra spp. antes y después de la aplicación de tres

\begin{tabular}{|c|c|c|}
\hline ACEFATO & & \\
\hline $\begin{array}{l}\text { CONCENTRACIÓN } \\
(\mathrm{g} / \mathrm{L})\end{array}$ & Antes & Después \\
\hline $0,5 \mathrm{~g} / \mathrm{L}$ & & \\
\hline $1,0 \mathrm{~g} / \mathrm{L}$ & & \\
\hline $1,5 \mathrm{~g} / \mathrm{L}$ & & \\
\hline
\end{tabular}

Fuente: Noemi Silva, 2016

Si se compara con el blanco del experimento, el ensayo realizado a la concentración más alta de Acefato evidencia la mayor pérdida de color, como puede verse en la Tabla 2.

Tabla 3. Registro de fotografías de Spirogyra spp. antes y después de la aplicación de tres dosis de Malatión.

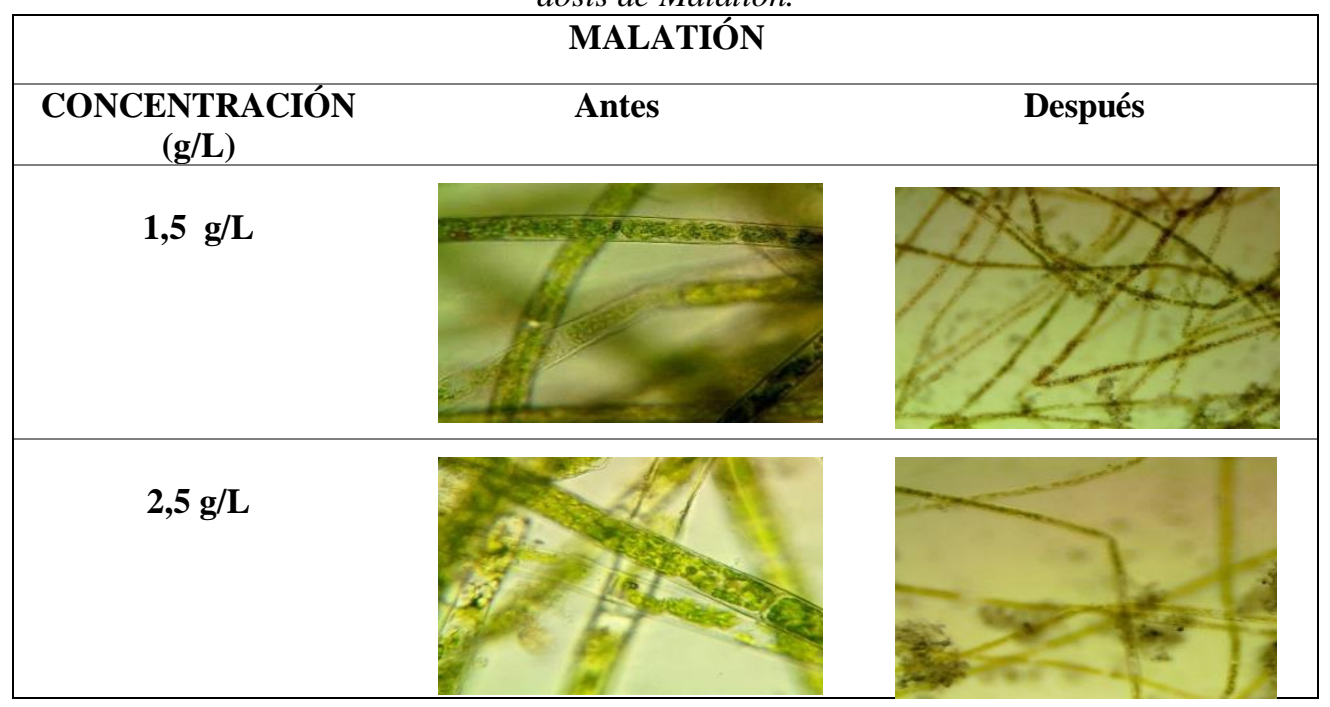




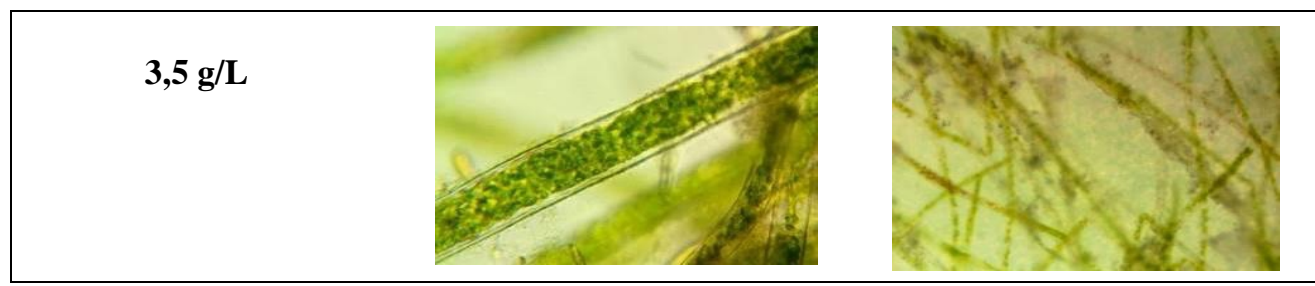

Fuente: Noemi Silva, 2016

En comparación con el blanco del experimento, los tres ensayos realizados a las tres concentraciones de Malatión evidenciaron que la coloración verde iba adquiriendo una tonalidad más oscura, como puede verse en la Tabla 3.

Tabla 4. Registro de fotografías de Spirogyra spp. antes y después de la aplicación de tres dosis de Curacrón.

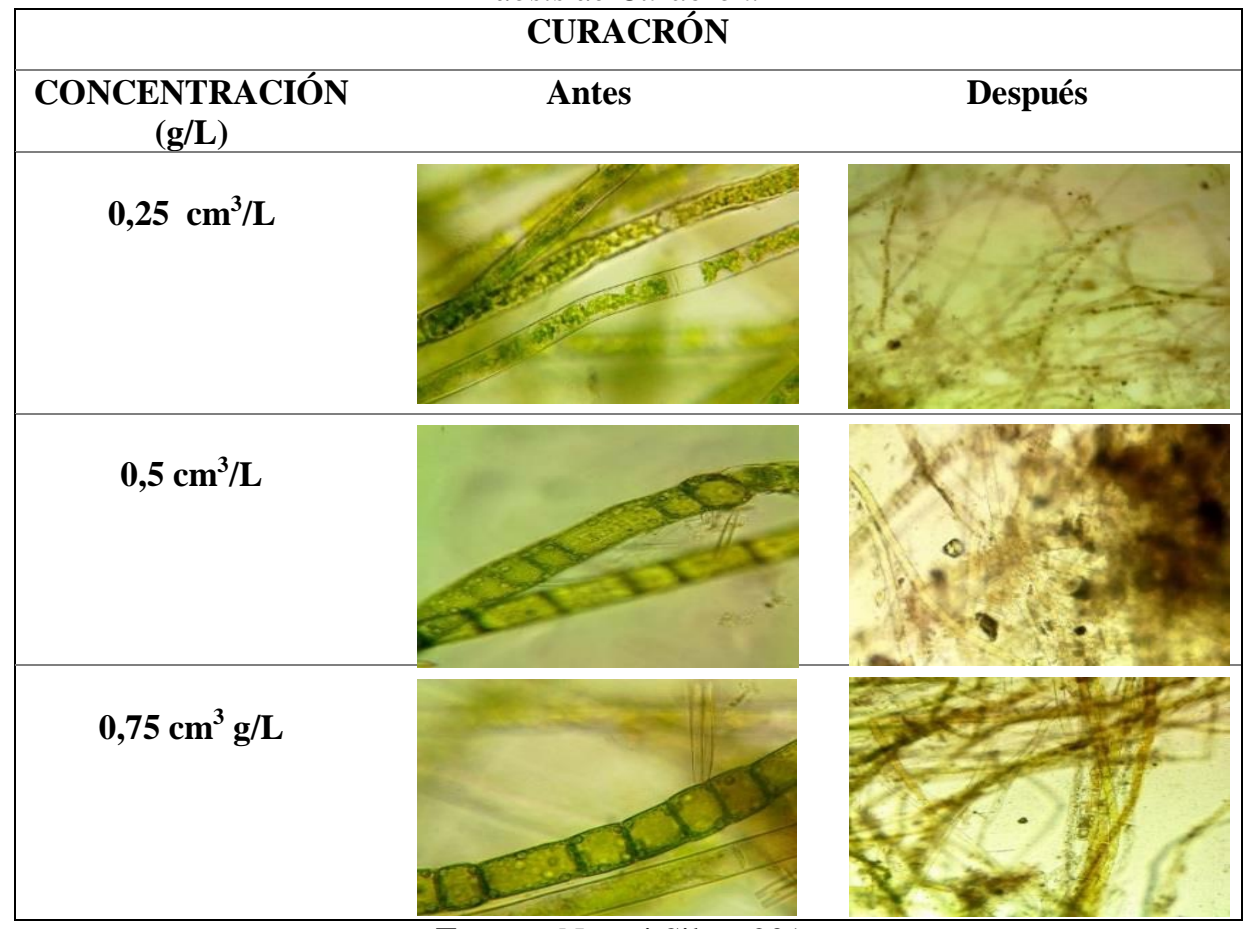

Fuente: Noemi Silva, 2016

Si se compara con el blanco del experimento, en el ensayo realizado a la concentración intermedia de Curacrón, así como a una dosis mayor, se evidencia la mayor pérdida de color, como puede verse en la Tabla 4. 
Tabla 5. Muestra testigo

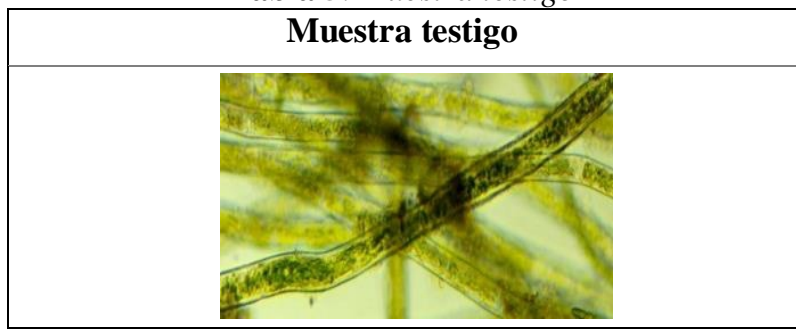

Fuente: Noemi Silva, 2016

La fotografía evidencia que la muestra testigo se mantuvo inmutable a lo largo del experimento (Tabla 5).

El cambio de coloración en la pigmentación coincide con lo reportado por Tandon, R. (1998), en la investigación "Interacción del endosulfán y el Malatión con algas azul-verdes Anabaena y Aulosira fertilissima" en donde se pudo evidenciar que Anabaena sobrevivió hasta $500 \mu \mathrm{g} / \mathrm{mL}$ pero blanqueó a mayor concentración del mismo. Aulosira resulto más sensible al Malatión ya que el alga perdió la capacidad de fijar nitrógeno, deteniéndose así su crecimiento.

\section{Resultados de medición de absorbancia}

Los resultados de la absorbancia medida en las muestras de agua y biomasa provenientes de las unidades experimentales después de haber aplicado los tres plaguicidas y medido a diferente rango de longitud de onda $(\lambda)$, (todos los rangos dentro del espectro en el que se puede medir clorofila) se detallan en las tablas 6,7 y 8 .

Tabla 6. Resultados de absorbancia, Spirogyra spp. + Acefato

\begin{tabular}{|cccc|}
\hline Tratamiento & & \multicolumn{2}{c|}{$\begin{array}{c}\text { Absorbancia (nm) } \\
\lambda:(449-533)\end{array}$} \\
\hline A1a & 0,40 & $\lambda:(534-616)$ & 0,47 \\
A1b & 0,33 & 0,17 & 0,42 \\
A1c & 0,39 & 0,15 & 0,47 \\
A2a & 0,60 & 0,38 & 0,68 \\
A2b & 0,57 & 0,36 & 0,66 \\
A2c & 0,59 & 0,35 & 0,64 \\
A3a & 0,58 & 0,25 & 0,55 \\
A3b & 0,53 & 0,22 & 0,59 \\
A3c & 0,52 & 0,23 & 0,62 \\
\hline Testigo A & 0,94 & 0,66 & 1,58 \\
\hline
\end{tabular}

Fuente: Silva, N. 2016 
Se aprecia una tendencia al decaimiento de la absorbancia con la variación de la concentración de cada plaguicida en todos los rangos de longitud de onda como se evidencia en la Tabla 6; denota el descenso general en la concentración de clorofila si se compara con el testigo A para el caso de los ensayos con Acefato, de manera especial la absorbancia medida en el rango de $\lambda$ : (449- 533) muestra una mayor disminución.

Tabla 7. Resultados de absorbancia, Spirogyra spp. + Malatión

\begin{tabular}{|cccc|}
\hline Tratamiento & $\lambda:(617-699)$ & $\begin{array}{c}\text { Absorbancia (nm) } \\
\lambda:(534-616)\end{array}$ & $\lambda:(449-533)$ \\
\hline B1a & 0,65 & 0,46 & 1,30 \\
B1b & 0,64 & 0,48 & 1,31 \\
B1c & 0,70 & 0,47 & 1,35 \\
B2a & 0,60 & 0,52 & 0,86 \\
B2b & 0,61 & 0,56 & 0,80 \\
B2c & 0,59 & 0,55 & 0,82 \\
B3a & 0,96 & 0,64 & 0,55 \\
B3b & 0,92 & 0,66 & 0,58 \\
B3c & 0,90 & 0,64 & 0,59 \\
\hline Testigo B & 0,98 & 0,65 & 1,56 \\
\hline
\end{tabular}

Fuente: Silva, N. 2016

Se aprecia una disminución general en la absorbancia medida para todos los ensayos realizados con el Malatión. La absorbancia medida en el rango de $\lambda$ : (449- 533) en particular muestra una mayor disminución al comparar con el testigo B del ensayo

Tabla 8. Resultados de absorbancia, Spirogyra + Curacrón

\begin{tabular}{|c|c|c|c|}
\hline \multirow[t]{2}{*}{ Tratamiento } & \multicolumn{3}{|c|}{ Absorbancia (nm) } \\
\hline & $\lambda:(617-699)$ & $\lambda:(534-616)$ & $\lambda:(449-533)$ \\
\hline C1a & 0,48 & 0,26 & 0,84 \\
\hline $\mathrm{C} 1 \mathrm{~b}$ & 0,46 & 0,22 & 0,80 \\
\hline $\mathrm{C} 1 \mathrm{c}$ & 0,46 & 0,23 & 0,83 \\
\hline $\mathrm{C} 2 \mathrm{a}$ & 0,12 & 0,06 & 0,23 \\
\hline $\mathrm{C} 2 \mathrm{~b}$ & 0,10 & 0,05 & 0,20 \\
\hline $\mathrm{C} 2 \mathrm{c}$ & 0,09 & 0,04 & 0,20 \\
\hline $\mathrm{C} 3 \mathrm{a}$ & 0,10 & 0,11 & 0,13 \\
\hline $\mathrm{C} 3 \mathrm{~b}$ & 0,08 & 0,12 & 0,14 \\
\hline $\mathrm{C} 3 \mathrm{c}$ & 0,12 & 0,13 & 0,12 \\
\hline Testigo C & 0,95 & 0,68 & 1,52 \\
\hline
\end{tabular}

Fuente: Silva, N. 2016 
En el caso de los ensayos realizados con Curacrón se evidencia una disminución de la absorbancia conforme aumenta la concentración del plaguicida; en este caso la comparación se realizó con el testigo C.

\section{Resultados de análisis de varianza y pruebas de Tukey}

Los resultados de análisis de varianza y pruebas de Tukey al 5\% se muestran en las tablas 9 a 14.

Tabla 9. Análisis de varianza para absorbancia a una $\lambda=617$ a $699 \mathrm{~nm}$

\begin{tabular}{|c|c|c|c|c|c|}
\hline F.V. & SC & $\begin{array}{l}\text { gl } \\
\text { valor }\end{array}$ & $\mathbf{C M}$ & $\mathbf{F}$ & p- \\
\hline Tratamiento & 2,88 & 9 & 0,25 & 415,65 & $<0,0001 * *$ \\
\hline Error & 0,01 & 20 & 0,00062 & & \\
\hline Total & 2,29 & 29 & & & \\
\hline
\end{tabular}

$p<0,05 Y<0,01 * *$

$p<0,05 Y>0,01 *$

$p>0,05 Y>0,01 n s$

En el análisis de varianza para absorbancia a una $\lambda=617$ a $699 \mathrm{~nm}$ (Tabla 9), se presentan diferencias altamente significativas para tratamientos con un p-valor $<0,0001^{* *}$, y un coeficiente de variación de 4,64\%.

Tabla 10. Prueba de Tukey al 5\% para absorbancia a una $\lambda=617$ a $699 \mathrm{~nm}$

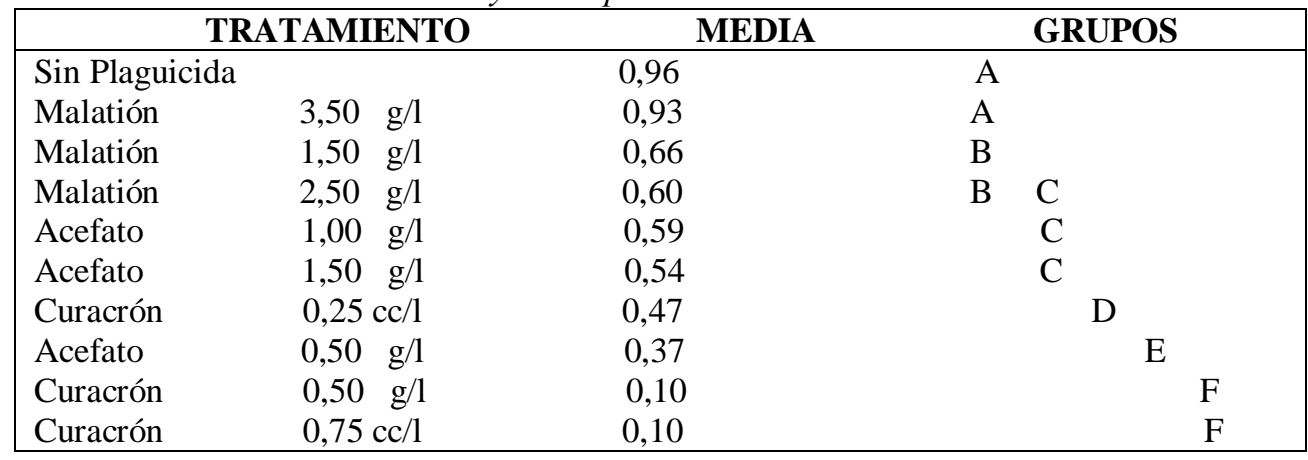

En la prueba de Tukey para absorbancia a una $\lambda=617$ a $699 \mathrm{~nm}$ (Tabla 10), se observa que el testigo y el Malatión en una dosis 3,50 g/l comparten el grupo " $\mathrm{A}$ " con valores de 0,96 y 0,93 respectivamente, en cambio los Curacron con dosis de 0,50 y $0,75 \mathrm{cc} / 1$ se encuentran en el grupo "F" con valores de 0,10 cada uno. Se puede apreciar que en esta longitud de onda existe una tendencia a la degradación de los pigmentos fotosintéticos presentes por decaimiento de la concentración de la clorofila en Spirogyra spp., disminuyendo el pigmento de acuerdo al tipo de plaguicida y dosis utilizada en comparación con el testigo mismo que mantiene un valor más alto de clorofila activa. 
Estos resultados coinciden con trabajos realizados por Iannacone, J. y Gutiérrez, A. (1999), en la investigación "Eco toxicidad de los agroquímicos Lindano y Clorpirifos sobre el nematodo Panagrellus, la micro alga Chlorella y el ensayo con Allium-test" en donde se concluye que la fotosíntesis neta y bruta y la respiración de la micro alga se ve afectada al ser expuesta a los agroquímicos mencionados.

Tabla 11. Análisis de varianza para absorbancia a una $\lambda=534$ a $616 \mathrm{~nm}$

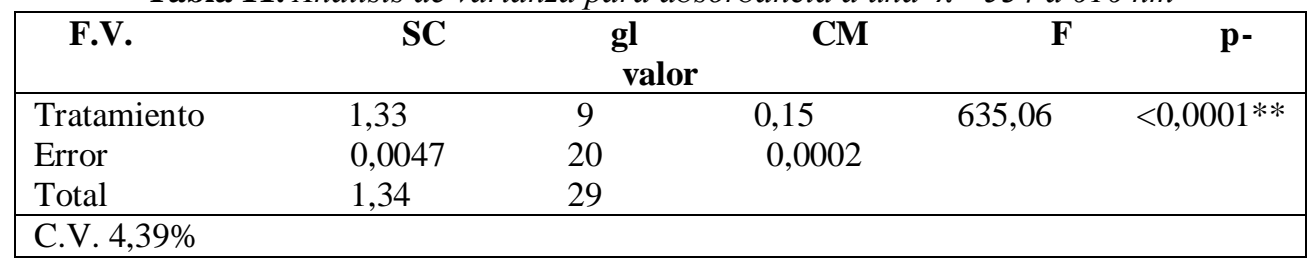

$p<0,05 Y<0,01 * *$

$p<0,05 Y>0,01 *$

$p>0,05 Y>0,01 n s$

En el análisis de varianza para absorbancia a una $\lambda=534$ a $616 \mathrm{~nm}$ (Tabla 11), se presentan diferencias altamente significativas para tratamientos con un p-valor $<0,0001^{* *}$, y un coeficiente de variación de 4,39\%.

Tabla 12. Prueba de Tukey al 5\% para absorbancia a una $\lambda=534$ a $616 \mathrm{~nm}$

\begin{tabular}{|c|c|c|c|c|c|c|c|}
\hline \multicolumn{2}{|c|}{ TRATAMIENTO } & \multicolumn{3}{|c|}{ MEDIA } & & \multicolumn{2}{|c|}{ GRUPOS } \\
\hline Sin Plaguicida & & 0,66 & $\mathrm{~A}$ & & & & \\
\hline Malatión & $3,50 \mathrm{~g} / 1$ & 0,65 & A & & & & \\
\hline Malatión & $2,50 \mathrm{~g} / 1$ & 0,54 & & B & & & \\
\hline Malatión & $1,50 \mathrm{~g} / \mathrm{l}$ & 0,47 & & & $\mathrm{C}$ & & \\
\hline Acefato & $1,00 \mathrm{~g} / 1$ & 0,36 & & & & $\mathrm{D}$ & \\
\hline Curacrón & $0,25 \mathrm{cc} / 1$ & 0,24 & & & & & $\mathrm{E}$ \\
\hline Acefato & $1,50 \mathrm{~g} / 1$ & 0,23 & & & & & E \\
\hline Acefato & $0,50 \mathrm{~g} / 1$ & 0,16 & & & & & \\
\hline Curacrón & $0,75 \mathrm{cc} / 1$ & 0,12 & & & & & \\
\hline Curacrón & $0,50 \mathrm{cc} / 1$ & 0,05 & & & & & \\
\hline
\end{tabular}

En la prueba de Tukey para absorbancia a una $\lambda=534$ a $616 \mathrm{~nm}$ (Tabla 12), se observa que el testigo y el Malatión en una dosis 3,50 g/l comparten el grupo "A" con valores de 0,66 y 0,65 respectivamente; en cambio los Curacrón con dosis de $0,50 \mathrm{cc} / 1$ se encuentran en el grupo " $\mathrm{G}$ " con un valor de 0,05 .

El Acefato, Malatión y Curacrón actúan directamente sobre el alga en función de la concentración de cada plaguicida, y cada plaguicida afecta de manera diferente en la morfología y estructura física del alga. La clorofila puede ser medida a través de una espectrofotometría UV y visible en rangos de longitud de onda que van desde los 450 hasta los $700 \mathrm{~nm}$.

Estos resultados coinciden con trabajos realizados por Azizullah, P. (2011), en el estudio "Toxicidad comparativa de los pesticidas carbofuran y 
malathion al flagelado de agua dulce Euglena gracilis”, donde uno de los parámetros evaluados fue la eficiencia fotosintética y la concentración de pigmentos que recogen la luz. Este estudio evidenció que en experimentos a corto plazo hay afectaciones especialmente en la velocidad de natación del alga evaluada y mientras aumentaba la exposición los efectos se incrementaban también.

Tabla 13. Análisis de varianza para absorbancia a una $\lambda=449$ a $533 \mathrm{~nm}$

\begin{tabular}{|lccccc|}
\hline \multicolumn{1}{|c}{ F.V. } & $\mathbf{S C}$ & gl & $\mathbf{C M}$ & $\mathbf{F}$ & p- \\
& & valor & & & \\
\hline Tratamiento & 5,39 & 9 & 0,60 & 925,75 & $<0,0001^{* *}$ \\
Error & 0,01 & 20 & 0,0006 & & \\
Total & 5,41 & 29 & & & \\
\hline C.V. $3,56 \%$ & & & & & \\
$p<0,05 Y<0,01 * *$ & & & & \\
$p<0,05 Y>0,01 *$ & & & & \\
$p>0,05 Y>0,01$ ns & & & & & \\
& & & & & \\
\end{tabular}

En el análisis de varianza para absorbancia a una $\lambda=449$ a $533 \mathrm{~nm}$ (Tabla 13), se presentan diferencias altamente significativas para tratamientos con un $\mathrm{p}$-valor $<0,0001^{* *}$, y un coeficiente de variación de $3,56 \%$.

Tabla 14. Prueba de Tukey al $5 \%$ para absorbancia a una $\lambda=449$ a $533 \mathrm{~nm}$

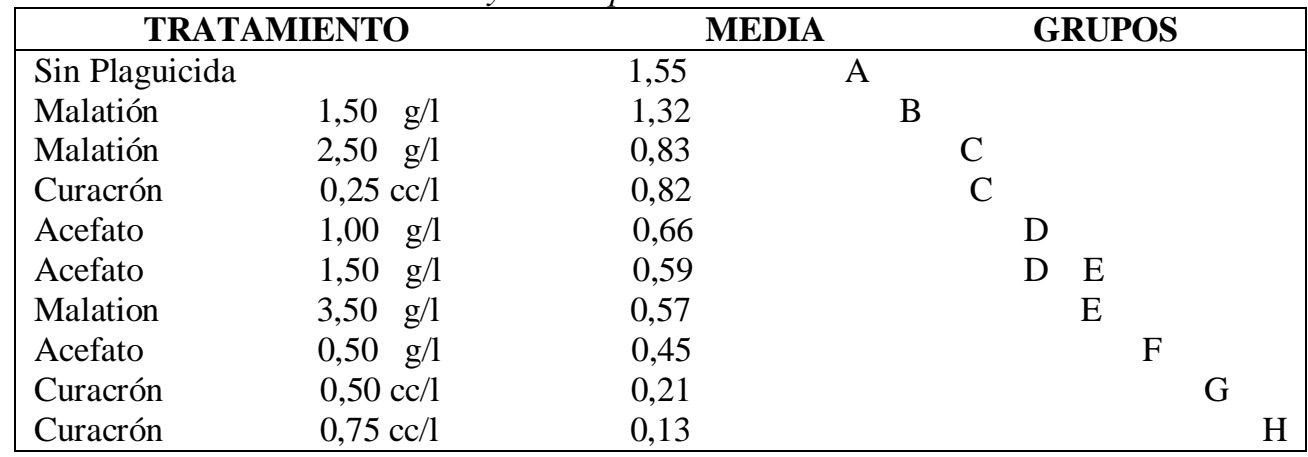

En la prueba de Tukey para absorbancia a una $\lambda=449$ a $533 \mathrm{~nm}$ (Tabla 14), se observa que el testigo con valor de 1,55 se ubica en el grupo "A", en cambio los Curacrón con dosis de $0,75 \mathrm{cc} / 1$ se encuentran en el grupo " $\mathrm{H}$ " con un valor de 0,13 .

El mecanismo de acción de degradación se fundamenta en la destrucción de las paredes celulares de las células pigmentadas de las algas y posterior pérdida de la coloración, coincidiendo con el trabajo realizado con Silva, $\mathrm{N}$ en el año 2016. En este estudio se observó que la acción de los plaguicidas organofosforados sobre los cloroplastos de las algas que son los organelos que albergan la clorofila también se incrementa en función del tiempo de exposición ya que en las primeras horas del experimento las algas 
permanecen intactas, mientras que al finalizar la etapa experimental (72 horas después) su deterioro es evidente.

Estos resultados también coinciden con el estudio realizado por Asselborn, V. et al. (2000), sobre "Efectos del insecticida organofosforado clorpirifos sobre el crecimiento y morfología de Selenastrum capricornutum Printz (Chlorophyta)", cuyo objetivo fue evaluar los efectos de distintas concentraciones del mencionado insecticida sobre el crecimiento y morfología de la micro alga. Se evidenció que a bajas concentraciones se inhibe el crecimiento del alga y a altas concentraciones hubo inhibición total, caso en el cual el químico no se considera alguicida sino sólo algistático ya que al cambiar de medio de cultivo limpio recuperaron su crecimiento. Las algas expuestas mostraron una marcada despigmentación y otros cambios morfológicos como aumento de tamaño y formación de gránulos.

\section{Conclusiones}

$\checkmark$ La acción de los plaguicidas organofosforados Malatión, Curacrón y Acefato sobre Spirogyra spp., provocó alteraciones paulatinas de la pigmentación verde natural de la biomasa algal. Al finalizar la etapa de exposición (72 horas) se evidenció la mayor afectación, de manera especial el Acefato degrada la pigmentación de Spirogyra.

$\checkmark$ En los ensayos realizados con Acefato y Malatión, existe un descenso general en los valores de absorbancia al ser comparados con los respectivos testigos. Estos valores muestran que, al ser comparada con los blancos utilizados, en el caso de la absorbancia medida a las muestras provenientes de los ensayos con Curacrón, la misma baja conforme la concentración de plaguicida aumenta.

$\checkmark$ Las pruebas de Tukey al 5\%, muestran que los tres plaguicidas utilizados influyen de manera directa en la pigmentación de las algas ya que actúan directamente sobre Spirogyra spp. en función de la concentración de cada plaguicida. De la misma manera, cada plaguicida afecta de manera diferente en la morfología y estructura física del alga.

$\checkmark$ Spirogyra spp. tiene gran potencial bioindicador de estos tres plaguicidas ya que existen claros cambios físicos y disminución de concentración de clorofila al ser expuesta a los mismos. De esta manera se evidencia una interacción estímulo-respuesta del alga frente al contacto con los plaguicidas ensayados, brindando a los agricultores de la comunidad de San Antonio la posibilidad de detectar tempranamente la presencia de estos contaminantes en sus fuentes de agua y evitar problemas mayores derivados de los plaguicidas. 


\section{Agradecimientos}

Al equipo científico de la Escuela Superior Politécnica de Chimborazo, por su valiosa contribución científica y técnica.

\section{References:}

1. Asselborn, V., Zolocar, Y. \& Parodi, E. (2000). Efectos del insecticida organofosforado clorpirifos sobre el crecimiento y morfología de Selenastrum capricornutum Printz (Chlorophyta). Rev. Comunicaciones científicas y Tecnológicas. Argentina.

2. Azizullah, R. (2011). Comparative toxicity of the pesticides carbofuran and malathion to the freshwater flagellate Euglena gracilis, Rev. Ecotoxicology. Inglaterra.

3. Cronquist, A. (1984). Introducción a la Botánica. 2a ed. México: Compañía Editorial Continental, pp. 56-72

4. Di Rienzo, J.A.C. F. (2017). InfoStat versión 2017. Grupo InfoStat, FCA, Universidad Nacional de Córdoba, Argentina. Obtenido de URL http:// www.infostat.com.ar

5. García, C. et al. (2012). "Biosensores Microalgales para la Detección de Contaminantes Ambientales: Una Revisión”. RCCV (Revista Complutense de Ciencias Veterinarias). [en línea], España. pp. 51-67. [Consulta: 24 mayo 2016]. ISSN 1988-2688. Disponible en: http://revistas.ucm.es/index.php/RCCV/article/view/39038/37663

6. González, J. et al. (2017). Evaluación de Spirogyra spp. como potencial bioindicador de plaguicidas organofosforados. Rev. Perspectiva 18(4). Perú.

7. González, C. et al. (2014) [Internet]. Bioindicadores: Guardianes de nuestro futuro ambiental. México. [Citado el 19 de agosto de 2016]. Disponible en: http://www.academia.edu /15617 373/ BIOINDICADORES_Guardianes_de_nuestro_futuro_ambiental

8. Iannacone, J. \& Gutiérrez, A. (1999). Ecotoxicidad de los agroquímicos Lindano y Clorpirifos sobre el nematodo Panagrellus, la microalga Chlorella y el ensayo con Allium. Rev. Agricultura técnica. Chile.

9. Lindao Córdova, V. A. (2017). Impacto en los niveles de colinesterasa en agricultores de tomate (Solanum lycopersicum L.) en la localidad de San Luis, Chimborazo por efecto del uso de insecticidas organofosforados y carbamatos.

10. Mariño, D. (2005). Determinación de residuos de plaguicidas organofosforados en el cultivo de mora (Rubus Glaucus) en dos cantones de la Provincia de Tungurahua Sangolqui- Ecuador. [Tesis]. Escuela Politécnica del Ejercito "GRAD. CARLOMAGNO 
ANDRADE PAREDES". Facultad de Ingeniería de Ciencias Agropecuarias.

11. Narsi, R. et al. (2007). Biosorption of Cr (III) from aqueous solution using algal biomass Spirogyra spp. ELSEVIER. Junio. 145: 142-147.

12. Orta, L. (2002). Contaminación de las aguas por Plaguicidas Químicos [Internet].Fitosanidad. Cuba. [Citado el 25 de junio de 2016]. Disponible en: http://www.redalyc.org/pdf/2091/209118292006.pdf

13. Peña, E. et al. (2006). Algas como indicadoras de contaminación. Colombia.

14. Robledo, D. (2001). Cultivo, adaptación morfológica y fisiopatología de macroalgas marinas de interés industrial. [tesis] Biblioteca virtual Miguel de Cervantes. España. 2001

15. Sederiak, N. \& Huynh, M. (2006). Algae Identification Field Guide. Canadá.

16. Silva, N. (2016). Evaluación en el laboratorio de la sensibilidad de Spirogyra spp como bioindicador de plaguicidas organofosforados. [Tesis]. Escuela Superior Plitécnica de Chimborazo. Facultad de Ciencias.

17. Tandon, R., Lal, R. \& Narayana Rao, V.V. (1988). Interaction of Endosulfan and malathion with blue-green algae Anabaena and Aulosira fertilissima. Environ. Pollut.52: 1-9. https://doi.org/10.1016 /0269-7491(88)90103-0

18. Vázquez, G. et al. (2006) [Internet]. Bioindicadores como herramientas para determinar la calidad del agua. ContacoS. 2006 60(1): 41-48. [Citado el 25 de junio de 2016]. Disponible en: http://www.izt.uam.mx/newpage/contactos/anterior/n 60ne/Bioagua.pdf

19. Vergara, C. (2016). Descripción de métodos de recolección y conservación de Algas. Herbario de la Universidad de Panamá. s.f. [Citado el 10 de agosto de 2016]. Disponible en: www.inbio.ac.cr/web_herbarios/web/pdf/protocolo-macroalgas.pdf 